\title{
Correlações presentes entre danos bucomaxilofaciais e lesões corporais em mulheres: uma revisão de literatura
}

\author{
Correlations present in maxillofacial and physical \\ injuries in women: a literature review
}

\author{
Alini Chiaperini', André Luiz Bérgamo', Lívia Aguiar Bregagnolo', \\ Janete Cinira Bregagnolo², Marlívia Gonçalves De Carvalho Watanabe², \\ Ricardo Henrique Alves Da Silva ${ }^{3}$
}

Chiaperini A, Bérgamo AL, Bregagnolo LA, Bregagnolo JC, Watanabe MGC, Silva RHA. Correlações presentes entre danos bucomaxilofaciais e lesões corporais em mulheres: uma revisão de literatura. Saúde, Ética \& Justiça. 2008;13(2):72-8.

Resumo: A violência contra a mulher é um fenômeno social de alta complexidade e graves paradoxos, seja no aspecto ético, no cultural, no político, no religioso, como também nas tentativas de explicação pelas mais variadas correntes e tendências. É algo que a sociedade e a comunidade científica não podem desconsiderar. A presença da Odontologia Legal, no que tange às lesões do complexo maxilo-mandibular em mulheres, torna-se indispensável na constatação e reparação dos danos. O presente trabalho objetiva demonstrar, através de uma revisão da literatura, as diversas correlações existentes entre as lesões corporais, mais especificamente as relacionadas aos danos bucomaxilofaciais, em mulheres, dando embasamento teórico aos profissionais que atuam na prática pericial em Traumatologia Forense.

Descritores: Odontologia legal. Violência contra a mulher. Prova pericial. Traumatismos maxilofaciais. Traumatismos mandibulares.

INTRODUÇÃo

A violência contra a mulher é um fenômeno social de alta complexidade e graves paradoxos, seja no aspecto ético, no cultural, no político, no religioso, como também nas tentativas de explicação pelas mais variadas correntes e tendências.

Souza ${ }^{1}$ explica que, apesar da violência contra a mulher ser de grande abrangência coletiva, tornase difícil a intervenção em vista da dificuldade de explicação e de sua visibilidade.

Apesar da percepção de atos violentos por parte das mulheres e do aumento progressivo de queixas na Delegacia da Mulher, esse número não representa sua magnitude, pois muitas mulheres não denunciam seus agressores.

Segundo Minayo $^{2}$, a violência não faz parte da

\footnotetext{
1 Cirurgiões-Dentistas graduados pela Faculdade de Odontologia de Ribeirão Preto/USP.

2 Professoras Associadas - Faculdade de Odontologia de Ribeirão Preto/USP.

3 Professor Doutor - Faculdade de Odontologia de Ribeirão Preto/USP.
}

Endereço Para correspondência: Prof ${ }^{a}$. Dr ${ }^{a}$. Janete Cinira Bregagnolo. Avenida do Café, s/n, Bairro Monte Alegre, Ribeirão Preto, SP. CEP 14040-904. E-mail: janete @ forp.usp.br 
natureza humana e não tem raízes biológicas, pois se trata de um complexo e dinâmico fenômeno biopsicossocial, mas seu espaço de criação e desenvolvimento é a vida em sociedade.

A partir da década de 1990, a violência contra a mulher passa a ser considerada sob uma nova ótica, quando a Organização Pan-Americana de Saúde (OPAS) reconhece que a violência, pelo número de vítimas e a magnitude de seqüelas orgânicas e emocionais que produz, adquire um caráter endêmico e se converte num problema de saúde pública ${ }^{3}$.

A violência contra a mulher, além de ser um problema político-social, é algo que a sociedade e a comunidade científica não podem desconsiderar. Desse modo, a presença da Odontologia Legal, no que tange às lesões do complexo maxilomandibular em mulheres, torna-se indispensável, na constatação e reparação dos danos.

Desta forma, o presente trabalho objetiva demonstrar as diversas correlações existentes entre as lesões corporais, mais especificamente relacionadas aos danos bucomaxilofaciais, em mulheres, dando embasamento teórico aos profissionais que atuam na prática pericial em Traumatologia Forense.

\section{Correlação entre trauma e gênero}

Em 1990, o Instituto Brasileiro de Geografia e Estatística ${ }^{4}$ trouxe dados que possibilitam entender o fenômeno da violência doméstica, que ocorre com muito mais intensidade entre as mulheres $(63 \%)$ do que entre os homens (37\%). Isso mostra que a mulher é a grande vítima desse tipo de violência, e que o espaço doméstico constitui-se em um ambiente de risco ${ }^{5}$.

Segundo Tajden e Thoennes ${ }^{6}$, embora alguns homens sejam agredidos por suas parceiras mulheres, a maior parte da violência é contra as mulheres. Nos serviços de emergência, as ocorrências encontradas indicam que de $22 \%$ a $35 \%$ das mulheres sofrem algum tipo de violência ${ }^{7}$.

Heise et al. $^{8}$ verificaram que a violência física na vida adulta, vinda de um parceiro, atinge entre $20 \%$ e $50 \%$ das mulheres ao redor do mundo ao menos uma vez na vida. E estudos populacionais e em serviços indicam maior risco de agressão às mulheres por parte de pessoas próximas como parceiros e familiares, do que por estranhos ${ }^{6,8}$. E, ao contrário do que pensa o senso comum, o abuso acontece em mulheres de todas as idades, raças, religiões, independente do nível de instrução ${ }^{6,9-10}$.

Segundo Suarez e Bandira ${ }^{11}$, todos os tipos de barbaridades foram cometidos contra as mulheres, em virtude da desigualdade de gêneros. Nessa reflexão, Costa e Moura $^{12}$, seguindo a mesma linha de pensamento, referem que "o patriarcado institui e legitima a violência contra a mulher, não apenas no aspecto material e físico, mas também e, principalmente, na dimensão psicológica e subjetiva".

Nesse sentido, o abuso pelo esposo, também determinado como violência pelo parceiro íntimo (IPV), é um comportamento violento que ocorre entre parceiros em um relacionamento íntimo, independentemente do estado civil. Injúrias e traumas não-acidentais são um importante risco para saúde, com significante morbidade e mortalidade associadas a estas injúrias intencionais, sendo que dados americanos indicam mais de um milhão de mulheres e 150.000 homens vítimas de IPV todo ano $^{13}$.

Agressões a mulheres são tão freqüentes e presentes que as seqüelas associadas estão presentes nas providências de cuidados associados à saúde que têm se expressado como uma síndrome. A síndrome da mulher agredida foi definida como um complexo sintoma ocorrendo como um resultado de ações abusivas direcionadas à mulher por seu parceiro homem ${ }^{13}$.

Quanto aos agressores, na maioria dos casos é o próprio marido ou parceiro ou ainda o ex. 0 local de ocorrência é o espaço doméstico, pois 40 a $80 \%$ dos espancamentos de mulheres ocorrem no domicílio 5 .

Mais recentemente, a partir da década de 90, essa forma de violência vem sendo concebida como baseada nas relações de gênero e, também, como uma questão de saúde e de direitos humanos ${ }^{8}$.

\section{Correlação entre faiXa etÁRia e trauma}

De acordo com o Relatório Geral sobre a Mulher na Sociedade Brasileira ${ }^{14}$, nas faixas etárias em que a mulher está geralmente casada, o espancamento é demasiado freqüente, sobretudo entre os 18 e os 29 anos de idade, recaindo, constantemente, sobre as mesmas vítimas, demonstrando um grande risco de rotinização da violência para a mulher.

Estudos internacionais mostram alta prevalência do problema nos serviços de saúde. Pesquisa 
em atenção primária apresenta uma freqüência de $21,4 \%$ das mulheres relatando violência doméstica a partir dos 18 anos $^{15}$. Segundo o estudo de Le et al. ${ }^{16}$, em 236 casos de emergência por violência doméstica, atendidos entre os anos de 1992 e 1996 no Legacy Emanuel Hospital, EUA, a média de idade foi 31,4 anos. Já em trabalho de Schraiber et al. $^{17}$, com uma população de 322 mulheres no município de São Paulo, verificou-se que a idade média foi de 28 anos.

No estudo de Moura e Oliveira ${ }^{18}$, que trata da análise das percepções das mulheres vítimas de lesão corporal dolosa em relação à violência vivenciada, o campo da pesquisa teve como cenário a Delegacia Especial de Atendimento à Mulher, na região Oeste do município do Rio de Janeiro, onde a idade das mulheres variou entre 22 e 34 anos.

De acordo com os estudos de Ogundare et al. ${ }^{19}$, no Columbia General Hospital, Washington (EUA), foi realizado um levantamento entre os anos de 1990 e 1999, a faixa etária mais afetada foi a de 2534 anos (37,9\%), seguida de $35-44$ anos (32,2\%), e a população menos afetada foi a de 65 anos ou mais.

\section{Correlação entre trauma e etNia}

Scherer et al. ${ }^{20}$ afirmam que o maior número de traumatismos faciais ocorrem em indivíduos da raça negra, seguidos por brancos.

Em um estudo de Schraiber et al. ${ }^{17}$, realizado com uma população de 322 mulheres, no município de São Paulo, entre as usuárias de uma Unidade Básica de Saúde, a maioria das mulheres entrevistadas, vítimas de agressão física, considerava-se de cor branca $(47,8 \%)$ e morava com o companheiro na época da pesquisa (59,0\%).

Demograficamente, o estudo populacional de Ogundare et al. ${ }^{19}$ mostrou que $92,78 \%$ dos pacientes diagnosticados com fratura de mandíbula eram negros, e o grupo menos afetado foi de hispânicos, entre os anos de 1990 e 1999, no Columbia General Hospital, Washington (EUA).

\section{CorrelaÇão entre trauma E ClassificaÇão do tipo de DANO}

Os atos de violência física foram classificados conforme sua gravidade segundo estudo da Organização Panamericana de la Salud $^{3}$ em: a) Ato moderado: ameaças, desde que não relativas a abuso sexual e sem uso de armas; agressões contra animais ou objetos pessoais e violência física na forma de empurrões, tapas, beliscões, sem uso de quaisquer instrumentos perfurantes, cortantes ou que gerem contusões; b) Ato severo: agressões físicas com lesões temporárias; ameaças com uso de arma, agressões físicas com cicatrizes, lesões permanentes, queimaduras e uso de arma.

Segundo Schraiber et al. ${ }^{17}$, no município de São Paulo, entre usuárias de uma Unidade Básica de Saúde, verificou que em $63,3 \%$ dos casos as agressões físicas foram consideradas severas e, $36,7 \%$ foram classificadas como moderadas.

Lesões corporais simples ou de natureza leve são aquelas comprometidas na fórmula genérica do artigo 129 do Código Penal Brasileiro21, não acarretando qualquer dos resultados previstos nos parágrafos $1^{\circ}, 2^{\circ}$ e $3^{\circ}$, no entanto, o crime exige um mínimo indispensável a constituir verdadeiramente um dano à integridade corporal ou à saúde.

Sob o aspecto odontológico, nas lesões corporais de natureza leve concentram-se a grande maioria dos ferimentos: lesões nos tecidos moles, como gengiva e mucosas, as ligeiras luxações dentárias, as fraturas coronárias de pequena extensão, uma leve periodontite traumática ${ }^{22}$, ou seja, as lesões de menor 'monta', que não comprometam as funções mastigatórias em caráter permanente e que não acarretem maiores riscos ou recuperação demorada ${ }^{23}$.

As lesões consideradas graves, alinhadas no parágrafo $1^{\circ}$ do artigo 129 do Código Penal Brasileiro ${ }^{21}$, são aquelas que resultam em: incapacidade para ocupações habituais por mais de 30 dias; perigo de vida; debilidade permanente de membro, sentido ou função; aceleração de parto; já as lesões corporais de natureza gravíssima estão dispostas no parágrafo $2^{\circ}$ do mesmo artigo, sendo assim consideradas se resultarem em: incapacidade permanente para o trabalho; enfermidade incurável; perda ou inutilização de membro, sentido ou função; deformidade permanente; aborto.

\section{Correlação entre trauma e AGente etiológico do dano}

A violência pode ser definida como violação dos Direitos Humanos, pelo constrangimento da vontade. Do ponto de vista legal, encontramos a seguinte classificação de ato violento: de ordem física (infanticídio, homicídio, espancamento, se- 
qüestro e rapto); de ordem psicológica (chantagem, ameaça, humilhação e desprezo); de ordem moral (calúnia, difamação e injúria); de ordem sexual (estupro e ato libidinoso), sendo os últimos capazes de envolver todos os demais: físico, psicológico e moral24.

A quantidade de mulheres que denunciam o agressor é pequena com relação à extensão do problema, e isso ocorre, segundo Saffioti ${ }^{25}$, pelas mais diversas razões: uma delas é o fato de que a mulher geralmente tem uma relação de afeto com o agressor, outra é econômica e consiste no fato de que ao terminar o casamento, mesmo uma mulher que possua uma profissão, estará defasada com relação ao mercado de trabalho, inclusive no que se refere a salário, afinal, terá passado anos isolada do espaço público.

No Brasil, dados referentes à violência contra a mulher demonstram que $70 \%$ dos incidentes acontecem dentro de casa, onde o criminoso é o próprio marido ou companheiro da vítima. Mais de $40 \%$ dos abusos envolvem lesões corporais graves causadas por socos, tapas, chutes, armamentos e espancamentos, queimaduras dos seios e genitália, e estrangulamento ${ }^{26}$.

Segundo dados do Núcleo de Odontologia Legal (NOL) do Instituto Médico-Legal de São Paulo, no período entre 1993 e 1998, do total de casos de lesões corporais, a natureza do agente etiológico da agressão mais freqüente foram socos, pontapés e projéteis de arma de fogo ${ }^{27}$.

Le et al. ${ }^{16}$ verificaram em seu estudo que as injúrias foram causadas por meio de força brusca ou penetrante ou a combinação de ambas, sendo presente o uso de arma em 15\% dos casos, sendo a maioria destas armas objetos rombos (garrafas, paus, canos) ou faca.

Schraiber et al. ${ }^{17}$ observaram que o agressor mais identificado pelas mulheres entrevistadas foi o companheiro, seguido por familiares. Dos casos de agressão física perpetrada pelo companheiro, 78,0\% envolviam agressões severas. Kruger ${ }^{28}$ (1984) cita uma pesquisa realizada no Hospital Geral do Distrito de Colúmbia (EUA), com 540 casos de fratura de mandíbula, em que a violência física foi responsável por $69 \%$ das fraturas, os acidentes por $27 \%$ (incluindo os acidentes automobilísticos), a prática de esportes por $2 \%$ e processos patológicos por $4 \%$.

Segundo Ochs et al. ${ }^{29}, 23 \%$ dos traumas de cabeça e pescoço que não foram causados por acidente automobilístico foram resultado de violência doméstica e 94\% das vítimas de violência doméstica tinham cabeça ou pescoço traumatizados ou ambos.

\section{CorRelação entre trauma E descrição dAS Lesões}

Tipicamente, os traumas faciais são compostos por lesões de tecidos moles e fraturas ósseas. A mais significante prioridade no paciente com fratura facial é o descobrimento ou o parecer da ameaça de vida nas injúrias associadas ${ }^{30}$.

No estudo de Le et al. ${ }^{16}$, no qual as informações foram obtidas de todas as mulheres que relataram uma história positiva de lesões por seus esposos ou parceiros sexuais durante este período, as lesões foram reportadas de acordo com a localização anatômica da cabeça, maxilofacial, pescoço, seio, tórax, abdômen, costas, nádegas, e extremidades. As lesões foram também classificadas como lacerações, abrasões, fraturas e deslocamentos. As lesões faciais foram classificadas de acordo com localização, tipo, lado (direito ou esquerdo), e terço facial, e como resultado o autor encontrou um total de 257 contusões e abrasões, 70 lacerações, 93 fraturas e 93 fraturas com deslocamentos.

\section{Correlação entre trauma e região da face afetada}

Para Fávero ${ }^{31}$, a cabeça é a região do corpo mais freqüentemente comprometida pelos traumas. O acometimento freqüente da face nos casos de agressão ocorre porque esta é uma porção integrante da cabeça, uma região pouco protegida e usualmente exposta.

Berrios e Grady32 mostraram que 68\% das mulheres que foram agredidas por seus parceiros íntimos sofreram lesões de cabeça e pescoço, incluindo lacerações, contusão e fraturas. E, de acordo com o estudo realizado por Schraiber et al. ${ }^{17}$, as regiões do corpo mais atingidas foram: face $(28,0 \%)$; cabeça e pescoço $(26,6 \%)$; seguidos pelos membros superiores anteriores $(25,2 \%)$; membros inferiores anteriores (16,8\%); costas (16,8\%); barriga (14,0\%); tronco (8,4\%); membros superiores posteriores $(8,4 \%)$; seios $(8,4 \%)$; nádegas $(6,3 \%)$; e outras regiões $(9,8 \%)$.

Já no estudo realizado por Le et al. ${ }^{16}$ foi verificado que a maior parte das lesões foi localizada na face, sendo que $81 \%$ das vítimas tiveram presença de lesões maxilofaciais, $50 \%$ tiveram uma lesão maxilofacial isolada com somente a presença de 
Chiaperini A, et al. Correlações presentes entre danos bucomaxilofaciais e lesões corporais em mulheres.

trauma, demonstrando, ainda, que muitas mulheres vítimas de violência doméstica sofrem um alto número de traumas maxilofaciais.

Motamedi $^{33}$ observou, entre os anos de 1996 e 2001, em um centro médico com 237 pacientes: 173 fraturas mandibulares $(72,9 \%), 33$ maxilares $(13,9 \%), 32$ zigomática $(13,5 \%), 57$ zigomáticoorbital $(24,0 \%), 5$ cranial $(2,1 \%), 5$ nasal $(2,1 \%)$ e 4 injúrias frontais $(1,6 \%)$.

E Ramos ${ }^{23}$ constatou dentre os tipos de danos detectados nos exames de corpo de delito a existência de fraturas da porção coronária e/ou radicular de dentes, fraturas ósseas de mandíbula e maxila, perdas dentárias, luxações dentárias ou da articulação temporomandibular (ATM), desordens da ATM, anquilose da ATM e ferimentos em tecidos moles. Entre os dados, as fraturas de porção coronária e as perdas dentárias constituíram os danos mais freqüentes.

Correlação entre trauma e local de registro da OCORRÊNCIA

Goldberg $^{34}$, em relação ao funcionamento da Delegacia de Defesa da Mulher, diz que: “(...) o funcionamento faria transparecer, de forma explosiva, a violência ensurdecida contra a mulher. De acordo com o Decreto número 23.769 a essa delegacia, assim como às demais instaladas no Estado e demais regiões do país, caberia a investigação e apuração dos delitos ocorridos contra a pessoa do sexo feminino, relativos às lesões corporais e crimes contra os costumes, assim como a hierarquização de delitos e penas".
O descrito anteriormente tornou-se fato de relevância quando foram analisados os primeiros cinco meses de atuação desta primeira delegacia, registrando 2.037 casos de violência, um número considerável de ocorrências, situação preocupante ${ }^{18}$.

Soares $^{35}$ verificou que, no Brasil, os estudos de casos são, basicamente, os denunciados nas Delegacias de Defesa da Mulher e apresentam um padrão centrado na violência doméstica.

$\mathrm{Na}$ análise dos casos de maior incidência de violência contra mulheres, registrados na Delegacia Especial de Atendimento à Mulher (DEAM), na região Oeste (Rio-Oeste) do Estado do Rio de Janeiro, foi observado que quando a mulher chega a prestar queixa na DEAM e o inquérito é instaurado, em alguns casos ela continua recebendo ameaças do marido ou do companheiro. Verificou-se nesse estudo que, dentre as mulheres que procuraram a delegacia e foram entrevistadas, apenas três chegaram a prestar queixa na DEAM Rio-Oeste, enquanto as demais não o fizeram, por manterem um laço de afetividade com o agressor que se sobrepõe à violência ${ }^{18}$.

\section{Considerações finais}

Desta forma, verifica-se a abrangência da atuação da Traumatologia Forense, no âmbito da Odontologia Legal e da Medicina Legal, no que se refere ao diagnóstico e à classificação das lesões em danos bucomaxilofaciais, tendo em vista a prevenção e o combate a tais agressões, e o conhecimento das informações teóricas a fim de que se realize um bom trabalho na prática pericial.

Chiaperini A, Bérgamo AL, Bregagnolo LA, Bregagnolo JC, Watanabe MGC, Silva RHA. Correlations present in maxillofacial and physical injuries in women: a literature review. Saúde, Ética \& Justiça. 2008;13(2):72-8.

АвsтRAct: Violence against women is a highly complex social phenomenon with severe paradoxes, in ethical, political, or religious aspects, as well as regarding the attempts of explaining it through the most varied chains and tendencies. It is something that should not be disregarded by the society and scientific community. Legal Dentistry, in terms of the maxillo-mandibular complex injuries in women, becomes indispensable in identifying and repairing any harm. The purpose of this study is to show, by means of a literature review, the several existing correlations between physical injuries, more specifically regarding maxillofacial injuries, in women, thus providing a theoretical basis for professionals working with Forensic Traumatology.

KEYwORDs: Forensic dentistry. Violence against women. Expert testimony. Maxilofacial injuries. Mandibular injuries. 


\section{REFERÊNCIAS}

1. Souza VLC. A violência conjugal e sua influência na decisão da mulher pelo aborto [dissertação]. Salvador: Escola de Enfermagem da Universidade Federal da Bahia; 2000.

2. Minayo MCS. A violência social sob a perspectiva da saúde pública. Cad Saúde Públ. 1994;10(1):7-18.

3. OPAS - Organizacion Panamericana de la Salud. Primeira Conferencia Interamericana sobre sociedad, violencia y salud. Bol Epidemiol. 1993.

4. IBGE. Instituto Brasileiro de Geografia e Estatística. Justiça e vitimização: participação política-social. Rio de Janeiro: FIBGE; 1990.

5. Diniz SG. A violência de gênero como questão de saúde. Rede Saúde. 1997;(14):7-12.

6. Tjaden P, Thoennes N. Prevalence, incidence, and consequences of violence against women: findings from the national violence against women survey: research brief. 1998 [Cited 2008 July 2]. Available from: http://www.ncjrs.gov/pdffiles/172837.pdf.

7. American Medical Association. Diagnostic and treatment Guidelines on Domestic Violence. Arch Fam Med. 1992;1(1):39-47.

8. Heise L, Pitanguy J, Germain A. Violence against women. The hidden health burden. Washington: World Bank; 1994.

9. Jones RF, Horan DL. The American College of Obstetricians and Gynecologists: a decade of responding to violence against women. Int J Gynecol Obstet. 1997;58(1):43-50.

10. Frugoli UO. Avaliação dos danos do complexo maxilomandibular provocados por violência interpessoal: análise comparativa entre os pareceres odontológicos e os laudos médicos obtidos pelo Instituto Médico Legal de São Paulo nos anos de 1993 e 1998 [dissertação]. São Paulo: Faculdade de Odontologia da Universidade de São Paulo; 2000.

11. Suárez $M$, Bandira L. Violência, gênero e crime no Distrito Federal. Brasília: Paralelo 15; 1999.

12. Costa AM, Moura MAV. Aborto legal. Brasília: NESP/ CEAM/UNB; 1999.

13. U.S.C. Title 42- the Public Health and Welfare, Chapter 132 Victims of Child abuse, Subchapter IV- Reporting Requirements, 13031. Child abuse reporting, 6 January, 1997. Available from: http://uscode.house. gov/download/pls/42C132.txt. [Cited 2008 Aug 4].
14. IV Conferência Mundial sobre a Mulher. Relatório geral sobre a mulher na sociedade brasileira. Brasília; 1995.

15. McCauley J, Kern DE, Kolodner K, Dill L, Schrouder $\mathrm{AF}$, DeChant HK, et al. The "battering syndrome": prevalence and clinical characteristics of domestic violence in primary care internal medicine practices. Ann Intern Med. 1995;123(10):737-46.

16. Le BT, Dierks EJ, Ueeck BA, Homer LD, Potter BF. Maxillofacial injuries associated with domestic violence. J Oral Maxillofac Surg. 2001;59(11):127783.

17. Schraiber LB, D'Oliveira AFPL, França-Júnior IF, Pinho AA. Violência contra a mulher: estudo em uma unidade de atenção primária à saúde. Rev Saúde Públ. 2002;36(4):470-7.

18. Moura MAV, Oliveira PRF. A percepção das mulheres vítimas de lesão corporal dolosa. Escola Anna Nery Rev Enf. 2000; 4(2/3):257-67.

19. Ogundare BO, Bonnick A, Bayley N. Pattern of mandibular fractures in an urban major trauma center. J Oral Maxillofac Surg. 2003;61(6):713-8.

20. Scherer M, Sullivan WG, Smith-Jr DJ, Phillips LG, Robson MC. An analysis of 1423 facial fractures in 788 patients at an Urban Trauma Center. J Trauma. 1988;29(3):388-90.

21. Brasil. Decreto-Lei no 2.848, de 7 de dezembro de 1940: Código Penal Brasileiro. [Acesso em: 02 jul. 2008] Disponível em: http://www.planalto.gov.br/ CCIVIL/Decreto-Lei/Del2848compilado.htm.

22. Cardozo HF, Penna JB. Avaliação das lesões dentárias em âmbito penal. Tribuna Advogado. 1997;2(19):10.

23. Ramos DG. Contribuição para o estudo jurídico das lesões corporais que incidem sobre o complexo maxilo-mandibular [dissertação]. São Paulo: Faculdade de Odontologia da Universidade de São Paulo; 1998.

24. Aquino E, Franco I, Marinho L. Saúde, sexualidade e direitos reprodutivos: a situação das mulheres na Bahia. In: II Conferência Mundial da Mulher, 1995, Salvador.

25. Saffioti HIB. Violência doméstica ou a lógica do galinheiro In: Kupstas M. Violência em debate. São Paulo: Moderna; 1997. p.39-57.

26. Thomas $D Q$. Injustiça criminal: a violência contra a mulher no Brasil: um relatório do Americas Watch e do projeto dos Direitos das Mulheres. Nova York: 
Chiaperini A, et al. Correlações presentes entre danos bucomaxilofaciais e lesões corporais em mulheres.

Americas Watch; 1991.

27. NEV/USP. Núcleo de Estudos da Violência. $1^{\circ}$ Relatório nacional de direitos humanos. São Paulo: NEV/USP; 1998.

28. Kruger GO. Fraturas dos maxilares. In: Kruger GO. Cirurgia bucal e maxilofacial. 5a ed. Rio de Janeiro: Guanabara-Koogan; 1984. p.244-96.

29. Ochs HA, Neuenschwander MC, Dodson TB. Are head neck and facial injuries markers of domestic violence? J Am Dent Assoc. 1991;127(6):757-61.

30. Melo REVA, Freitas CM, Abreu TC. Trauma facial: Uma análise de 1316 pacientes. Rev Odontol Ciênc. 1996;11(21):167-81.

Artigo recebido em 24/06/2008. Aprovado em 03/09/2008.
31. Fávero F. Medicina legal. 9a ed. São Paulo: Martins; 1973.

32. Berrios DC, Grady D. Domestic violence: risk factors and outcomes. West J Med. 1991;155:133-5.

33. Motamedi MHK. An assessment of maxillofacial fractures: a 5-year study of 237 patients. J Oral Maxillofac Surg. 2003;61(1):61-4.

34. Goldenberg P, Medrado MA, Pasternostro MA. A violência contra a mulher: uma questão de saúde. In: Labra ME. Mulher saúde e sociedade no Brasil. Rio de Janeiro: Vozes; 1989.

35. Soares BM. Mulheres invisíveis: violência conjugal e novas políticas de segurança. Rio de Janeiro: Civilização Brasileira; 1999. 